\title{
Normal Forms and Configuration Singularities of a Space Manipulator
}

\author{
Krzysztof Tchon ${ }^{1}$ (D) . Witold Respondek ${ }^{2}$. Joanna Ratajczak ${ }^{1}$
}

Received: 30 November 2017 / Accepted: 24 May 2018 / Published online: 12 June 2018

(C) The Author(s) 2018

\begin{abstract}
This paper addresses the problem of normal forms and singularities of non-holonomic robotic systems represented by control-affine systems. By means of the concept of the end-point map of the system, and of the system's Jacobian, the configuration singularities have been defined as the control functions for which the Jacobian is not surjective. The presence of these singularities impairs performance of Jacobian motion planning algorithms. Being the singular optimal controls, the configuration singularities can be examined using the tools from the optimal control theory. The main idea of this paper is to rely the analysis of configuration singularities on normal forms of robotic systems. This idea has been applied to the dynamics of a space manipulator. Normal forms of this manipulator under the feedback equivalence have been obtained, and exploited in the analysis of its configuration singularities.
\end{abstract}

Keywords Non-holonomic robotic system $\cdot$ Motion planning $\cdot$ Singularities $\cdot$ Normal forms $\cdot$ Space manipulator

\section{Introduction}

The main reason for studying singularities of robots is that they impair the robot control, and specifically the operation of Jacobian motion planning algorithms. In order to better understand the kinematics singularities in manipulation robots (or holonomic robotic systems), some years ago we proposed the Normal Form Approach rooted in the singularity theory of functions $[1,2]$. The essence of that approach consists in providing a description of the robot kinematics around singularities by simple functions called normal forms. Because the equivalence between the original kinematics and their normal forms preserves singularities, it is possible to deduce the behaviour of the robot near singularities from the normal forms. In the area of mobile robots or, more generally, non-holonomic robotic systems subject

Research of authors no 1 and 3 was supported by the Wrocław University of Science and Technology under a statutory research project.

Krzysztof Tchoń

krzysztof.tchon@pwr.edu.pl

1 Chair of Cybernetics and Robotics, Electronics Faculty, Wroclaw University of Science and Technology, ul. Janiszewskiego 11/17, 50-372, Wroclaw, Poland

2 INSA de Rouen, Laboratoire de Mathématiques, Normandie Université, 76801, Saint-Etienne-du-Rouvray, France to Pfaffian constraints and represented by associated control systems, the role analogous to the kinematics is played by the end-point map. This map attaches the terminal point of time-evolution of the system's states (or of its outputs) to the control functions applied to the robotic system. A study of the end-point map is a leitmotif of the Endogenous Configuration Space Approach [3], and the singularities of the end-point map are called configuration singularities [4]. As in the holonomic robotic systems, at configuration singularities performance of Jacobian control and motion planning algorithms of the non-holonomic robotic systems deteriorates. For this reason comprehending singularities is crucial for efficient control.

Following these guidelines, in this paper we propose to characterize configuration singularities of non-holonomic robotic systems by normal forms, i.e. equivalent simple control systems whose behaviour at singularities is transparent. The equivalence relation employed here is the feedback equivalence of control systems that is known to preserve the singularities, and used in [5, 6]. For completeness of exposition we present a proof of this property in Section 6. What we examine are the singular extremals, singular controls, and singular curves of the normal form that, due to basic properties of the feedback transformation, can be converted into singular extremals, controls, and curves of the original system. This procedure will be applied and illustrated in detail with the example of a free-floating space manipulator subject to the non-zero linear and angular momentum 
conservation. First, three control system representations of the space manipulators are presented, and then four local normal forms (two for zero- and two for non-zero angular momentum) are derived along with the necessary feedback transformations, three of them mathematically corresponding to the forms of Darboux and Martinet [7]. The domains of existence of these normal forms are directly related with the kinematic singularities of the on-board manipulator or the dynamic singularities of the space manipulator [8]. Then, the singular extremals, singular controls, and singular trajectories for these normal forms are determined, using the tools available from the classic and the modern control theory [9-11], for a robotics perspective see [12]. Finally, on the basis of this result, we describe singular configurations of the space manipulator.

The contribution of this paper to robotics relies on introducing the normal form approach to study singularities of non-holonomic robotic systems, establishing normal forms under feedback for a space manipulator, together with explicit feedback transformations, and characterizing its configuration singularities. The space manipulator is represented by a control-affine system with 3 states and 2 inputs. A feedback classification of such systems under a genericity assumption has been accomplished in [7]. A novelty of our paper in this context consists in providing a normal form for a system that does not satisfy the genericity assumption (although it shows up at kinematic singularities of the onboard manipulator), and in designing explicitly the feedback transformation establishing the equivalence in all cases. The feedback classification that we provide implies that only two local behaviours of the manipulator may occur for non-zero angular momentum (one at regular positions and one at the above mentioned singularities) and only two local behaviours (at the same configurations, respectively) if the angular momentum vanishes.

This paper is composed in the following way. The next Section 2 introduces a control-affine system representation of non-holonomic robotic systems. Section 3 defines the end-point map and the system's Jacobian. The motion planning problem is described in Section 4, where we also explain the importance of non-singularity of the end-point map for Jacobian motion planning. Configuration singularities are characterized in Section 5. Section 6 is devoted to the feedback equivalence of control systems. The model of a space manipulator, its normal forms, and its configuration singularities are examined in Section 7. Section 8 concludes the paper.

\section{Control System Representation}

We shall study a robotic system described by generalized coordinates $q \in \mathbb{R}^{n}$ or, more generally, on an $n$-dimensional manifold $Q$, and subject to motion constraints in the affine Pfaffian form

$\mathcal{A}(q) \dot{q}=b(q)$.

The constraints are assumed independent, which means that the matrix $\mathcal{A}(q)$ has full row rank.

Affine Pfaffian constraints can be represented by an associated control-affine system of the form

$\dot{q}=f(q)+G(q) u=f(q)+\sum_{i=1}^{m} g_{i}(q) u_{i}$.

In Eq. 1 the drift vector field can be computed as $f(q)=$ $\mathcal{A}^{\#}(q) b(q)$, where $\mathcal{A}^{\#}(q)$ denotes a right inverse of $\mathcal{A}(q)$, and the control matrix $G(q)$ spans the null space of the Pfaffian matrix, i.e., the columns $g_{i}(q)$ of $G(q)$ are $m$ independent solutions of $\mathcal{A}(q) g_{i}(q)=0$. All these vector fields are assumed $C^{\infty}$ - smooth (which is always the case if $\mathcal{A}$ and $b$ are $C^{\infty}$ - smooth and $\mathcal{A}(q)$ is of full row rank). Notice that $\mathcal{A}^{\#}(q)$ is not unique because if $f(q)$ satisfies $\mathcal{A}(q) f(q)=b(q)$, then so does any $f(q)+$ $\sum_{i=1}^{m} \alpha_{i}(q) g_{i}(q)$, and therefore $f$ is given up to a feedback transformation. Similarly, the control vector fields $g_{i}$, annihilated by $\mathcal{A}$, are given up to an invertible transformation $g_{i}(q) \mapsto \sum_{i=1}^{m} \beta_{i}^{j}(q) g_{j}(q)$, where the matrix $\beta(q)=$ $\left(\beta_{i}^{j}\right)(q)$ is invertible. In the control-affine case, the velocities $\dot{q}$ respecting the constraints satisfy $\dot{q} \in \mathcal{F}(q(t))$, with

$\mathcal{F}=f+\mathcal{G}$

consisting of all $f+g$, where $g$ is any vector field in the distribution $\mathcal{G}$ spanned by the control vector fields $g_{i}$. Notice that $\mathcal{F}$ is an affine distribution and that applying a feedback transformation $u=\alpha(q)+\beta(q) \tilde{u}$ maps $f$ into $f+G \alpha=$ $f+\sum_{i=1}^{m} \alpha_{i} g_{i}$ and $G$ into $G \beta$, but preserves $\mathcal{F}$.

In the particular case when $b(q)=0$, we deal with the linear Pfaffian constraints

$\mathcal{A}(q) \dot{q}=0$.

It is well known that a system satisfying linear Pfaffian constraints can be represented by an associated driftless control system of the form

$\dot{q}=G(q) u=\sum_{i=1}^{m} g_{i}(q) u_{i}$,

which means that the velocities $\dot{q}$ respecting the linear constraints satisfy $\dot{q}(t) \in \mathcal{G}(q(t))$, where $\mathcal{G}$ is the distribution spanned by the vector fields $g_{i}$.

While the driftless representation (3) refers primarily to the kinematics of mobile robots or to the dynamics of space manipulators obeying the zero angular momentum condition, the control-affine system (1) describes the dynamics of mobile robots whose kinematics are subject to the d'Alembert principle or the dynamics of space manipulators conserving a non-zero angular momentum. Classical results 
in non-linear control assert that the driftless system is controllable if its control distribution $\mathcal{G}$ is bracket-generating. For the control systems (1) and (3) defined as representations of the Pfaffian constraints we shall study singular controls in the context of the motion planning problem.

\section{End-Point Map and Jacobian}

Given $T>0$, admissible control functions will be taken as Lebesgue square integrable functions of time defined on the interval $[0, T]$, taking values in $\mathbb{R}^{m}$. Together with the inner product $<u(\cdot), \tilde{u}(\cdot)\rangle=\int_{0}^{T} u^{T}(t) \tilde{u}(t) d t$ these control functions form the Hilbert space $\mathcal{H}=L_{m}^{2}[0, T]$. For a given $q_{0}$ and $u_{0}(\cdot) \in \mathcal{H}$ we fix $T>0$ such that the state trajectory $q(t)=\varphi_{q_{0}, t}(u(\cdot))$ of system (1), initialized at $q(0)=q_{0}$ and driven by $u_{0}(\cdot)$, exists on $[0, T]$. We define the end-point map $\mathcal{K}_{q_{0}, T}: \mathcal{U} \longrightarrow \mathbb{R}^{n}$ by

$\mathcal{K}_{q_{0}, T}(u(\cdot))=\varphi_{q_{0}, T}(u(\cdot))$,

where $\mathcal{U}$ is a neighbourhood of $u_{0}(\cdot)$ in $\mathcal{H}$. It is well known that this end-point map is continuously differentiable [13]. Its derivative

$J_{q_{0}, T}(u(\cdot))=\mathrm{D} \mathcal{K}_{q_{0}, T}(u(\cdot))$

is called the Jacobian of system (1). The value of the Jacobian at a given $v(\cdot) \in \mathcal{H}$ is computed by the linear approximation of system (1) along the input-state trajectory $(u(t)$, $q(t))=\left(u(t), \varphi_{q_{o}, t}(u(\cdot))\right)$ in the following way [3]. The linear approximation is a time-varying control system

$\dot{\xi}=A(t) \xi+B(t) v$

determined by the matrices

$A(t)=\frac{\partial(f(q(t))+G(q(t)) u(t))}{\partial q}, B(t)=G(q(t))$.

System (5) is controllable if and only if its controllability Gramian

$\mathcal{M}_{q_{0}, T}(u(\cdot))=\int_{0}^{T} \Phi(T, t) B(t) B^{T}(t) \Phi^{T}(T, t) d t$

has full rank $n$.

Having found the trajectory $\xi(\cdot)$ of the linear system (5) starting with $\xi(0)=0$, we get

$J_{x_{0}, T}(u(\cdot)) v(\cdot)=\xi(T)=\int_{0}^{T} \Phi(T, s) B(s) v(s) d s$,

where $\frac{\partial \Phi(t, s)}{\partial t}=A(t) \Phi(t, s), \Phi(s, s)=I_{n}$ denotes the fundamental matrix.

\section{Motion Planning}

In terms of the end-point map (4) the motion planning problem for system (1) can be stated in the following way. Given an initial state $q_{0} \in \mathbb{R}^{n}$ and a terminal point $q_{d} \in \mathbb{R}^{n}$, find a control function $u(\cdot) \in \mathcal{U}$ such that at $T$ the system's trajectory reaches $q_{d}$, i.e.

$q(T)=\mathcal{K}_{q_{0}, T}(u(\cdot))=q_{d}$.

We shall focus on Jacobian motion planning algorithms. Such an algorithm relies on solving the following implicit functional differential equation, see [3],

$J_{q_{0}, T}\left(u_{\theta}(\cdot)\right) u_{\theta}^{\prime}(\cdot)=-\gamma\left(K_{q_{0}, T}\left(u_{\theta}(\cdot)\right)-q_{d}\right)$,

where $u_{\theta}(t)$ denotes a differentiable curve contained in $\mathcal{H}$, parametrized by $\theta \in \mathbb{R}$, and ' refers to the differentiation with respect to a scalar parameter $\theta$. Assuming that a right inverse $J_{q_{0}, T}^{\#}(u(\cdot)): \mathbb{R}^{n} \longrightarrow \mathcal{U}$ of the Jacobian exists, the Eq. 10 transforms into the explicit form

$u_{\theta}^{\prime}(\cdot)=-\gamma J_{q_{0}, T}^{\#}\left(u_{\theta}(\cdot)\right)\left(K_{q_{0}, T}\left(u_{\theta}(\cdot)\right)-q_{d}\right)$.

Under assumption that the limit at $+\infty$ of the solution $u_{\theta}(t)$ of (11) exists, the control function $u_{d}(t)$ driving system (1) to $q_{d}$ is found as

$u_{d}(t)=\lim _{\theta \rightarrow+\infty} u_{\theta}(t)$

Differential Eq. 11 defines a Jacobian motion planning algorithm, hence any right Jacobian inverse determines such an algorithm. In order to compute a right Jacobian inverse for a fixed $u(\cdot) \in \mathcal{U}$, one needs, for any chosen $\eta \in \mathbb{R}^{n}$, to solve with respect to $v(\cdot) \in \mathcal{H}$ the Jacobian equation

$J_{q_{0}, T}(u(\cdot)) v(\cdot)=\eta$.

To get a unique solution, this equation may be included as an equality constraint into an optimal control problem in the linear approximation (5). For example, the Jacobian pseudoinverse (the Moore-Penrose generalized inverse) whose singularities may be regarded as a kind of canonical [14], comes from the control energy minimization

$\min _{\left\{v(\cdot) \mid J_{q_{0}, T}(u(\cdot)) v(\cdot)=\eta\right\}} \int_{0}^{T} v^{T}(t) v(t) d t$,

that results in a map $J_{q_{0}, T}^{J P \#}(u(\cdot)): \mathbb{R}^{n} \longrightarrow \mathcal{U}$, such that

$\left(J_{q_{0}, T}^{J P \#}(u(\cdot)) \eta\right)(t)=B^{T}(t) \Phi^{T}(T, t) \mathcal{M}_{q_{0}, T}^{-1}(u(\cdot)) \eta$,

where $\mathcal{M}_{q_{0}, T}(u(\cdot))$ is the controllability Gramian of system (5), given by Eq. 7. This inverse exists on condition that the controllability Gramian has full rank $n$. The motion planning algorithm corresponding to Eq. 14 is referred to as the Jacobian Pseudoinverse motion planning algorithm. Obviously, this algorithm is well defined under the condition that matrix (7) has full rank. This issue will be addressed in detail in the next section. 


\section{Configuration Singularities}

Configuration singularities of system (1) are defined as the control functions $u(\cdot) \in \mathcal{U}$ for which the Jacobian map $J_{q_{0}, T}(u(\cdot)): \mathcal{H} \longrightarrow \mathbb{R}^{n}$ is not surjective [4]. Otherwise, we refer to the configurations as regular. It results from Section 4 that a necessary and sufficient condition for $u(\cdot)$ to be a configuration singularity is that the rank of matrix (7) should be less than $n$. By definition, if $u(\cdot)$ is singular then the Jacobian motion planning algorithm (11) is not well defined, and one may expect that close to the configuration singularities its performance will deteriorate.

It follows that at regular configurations the linear approximation (5) is controllable, implying that the control-affine system (1) is locally controllable. Furthermore, it is well known that configuration singularities of system (1) coincide with its singular controls (see e.g. [10, 13]).

The concept of singular control has been incorporated into the optimal control theory since its beginning. To explain it, we shall associate with system (1) an adjoint variable $p^{T} \in$ $\left(\mathbb{R}^{n}\right)^{*}$, the dual space to $\mathbb{R}^{n}$, and define a Hamiltonian

$H(q, p, u)=p^{T}(f(q)+G(q) u)$.

A triple $(u(t), q(t), p(t)), p(t) \neq 0$ is called a singular extremal of this Hamiltonian if it satisfies the Hamilton's canonical equations and the optimality condition, i.e.,

$\dot{q}=\frac{\partial H(q, p, u)}{\partial p}, \dot{p}=-\frac{\partial H(q, p, u)}{\partial q}, \frac{\partial H(q, p, u)}{\partial u}=0$.

A control $u(t)$ giving rise to a singular extremal is called a singular control of system (1). The pair of functions of time $(u(t), q(t))$ is referred to as a singular control-state trajectory, while the plot of $q(t)$ in $\mathbb{R}^{n}$ forms a singular curve.

Specifically, for the control-affine system (1) a singular extremal $(u(t), q(t), p(t))$ should fulfil the following equations

$\dot{q}=f(q)+G(q) u, \quad \dot{p}^{T}=-p^{T} A, \quad p^{T} B=0$

and, additionally, $p^{T} f(q)=$ const, that makes the Hamiltonian constant along the singular extremal. The matrices $A$ and $B$ being given by Eq. 6 , we can re-write Eq. 15 as

$$
\begin{aligned}
& \dot{q}=f(q)+G(q) u, \quad \dot{p}^{T}=-p^{T} \frac{\partial(f(q)+g(q) u)}{\partial q}, \\
& p^{T} G(q)=0 .
\end{aligned}
$$

Since configuration singularities coincide with singular controls, (15) provides us with an efficient tool for detecting these singularities, in particular, in robotic systems. However, checking conditions (15) may be quite involved, so we propose to use to this aim normal forms of the system. The normal form is a simple control system equivalent to the original system. In this paper the equivalence will be understood as the feedback equivalence. It will be introduced in the next section, and then, in Section 7, the feedback equivalence and suitable normal forms will be applied to calculating configuration singularities of a space manipulator.

\section{Feedback Equivalence of Control Systems}

Consider a pair of control-affine systems

$\Sigma: \dot{q}=f(q)+G(q) u, \quad \tilde{\Sigma}: \dot{\tilde{q}}=\tilde{f}(\tilde{q})+\tilde{G}(\tilde{q}) \tilde{u}$,

where $q, \tilde{q} \in \mathbb{R}^{n}$ and $u, \tilde{u} \in \mathbb{R}^{m}$. These systems are called feedback equivalent, if there exist a smooth globally invertible change of coordinates $\tilde{q}=\varphi(q)$ and a feedback $u=\alpha(q)+\beta(q) \tilde{u}$, given by a smooth $\mathbb{R}^{m}$-valued function $\alpha$ and a smooth invertible $m \times m$ matrix $\beta(q)$, such that

$$
\begin{aligned}
& \frac{\partial \varphi}{\partial q}(q)(f(q)+G(q) \alpha(q))=\tilde{f}(\varphi(q)), \\
& \frac{\partial \varphi}{\partial q}(q) G(q) \beta(q)=\tilde{G}(\varphi(q)) .
\end{aligned}
$$

This equivalence is referred to as local, if $\varphi(q), \alpha(q)$, and $\beta(q)$ are defined locally only.

The feedback equivalence aids solving some control problems, like the trajectory tracking and stabilizability problems. For the present paper its crucial property is that it preserves configuration singularities. This observation is the starting point for the powerful and elegant theory of feedback invariants, see $[5,6]$, and can be formulated as follows.

Proposition 1 Feedback equivalence preserves configuration singularities. More precisely, if $(u(t), q(t), p(t))$ is a singular extremal for $\Sigma$, which is (locally) feedback equivalent to $\tilde{\Sigma}$ via $\tilde{q}=\varphi(q), u=\alpha(q)+\beta(q) \tilde{u}$, then $(\tilde{u}(t)$, $\tilde{q}(t), \tilde{p}(t))$ is a singular extremal for $\tilde{\Sigma}$, where

$$
\begin{aligned}
& u(t)=\alpha(q(t))+\beta(q(t)) \tilde{u}(t), \\
& \tilde{q}(t)=\varphi(q(t)), \\
& p^{T}(t)=\tilde{p}^{T}(t) \frac{\partial \varphi}{\partial q}(q(t)) .
\end{aligned}
$$

Proof We shall show that for any triple $(u(t), q(t), p(t))$ satisfying (16), i.e., any singular extremal of $\Sigma$, the corresponding triple $(\tilde{u}(t), \tilde{q}(t), \tilde{p}(t))$ satisfies

$$
\begin{aligned}
& \dot{\tilde{q}}=\tilde{f}(\tilde{q})+\tilde{G}(\tilde{q}) \tilde{u}, \dot{\tilde{p}}^{T}=-\tilde{p}^{T} \frac{\partial(\tilde{f}(\tilde{q})+\tilde{g}(\tilde{q}) \tilde{u})}{\partial \tilde{q}}, \\
& \tilde{p}^{T} \tilde{G}(\tilde{q})=0,
\end{aligned}
$$

and $\tilde{p}^{T} \tilde{f}=$ const , and thus is a singular extremal of $\tilde{\Sigma}$.

We have

$$
\begin{aligned}
\dot{\tilde{q}} & =\frac{\partial \varphi}{\partial q} \dot{q}=\frac{\partial \varphi}{\partial q}(f+G u)=\frac{\partial \varphi}{\partial q}(f+G \alpha+G \beta \tilde{u}) \\
& =\tilde{f}+\tilde{G} \tilde{u},
\end{aligned}
$$


proving that the first equation of Eq. 16 is mapped into the first equation of Eq. 19.

Then calculate

$$
\begin{aligned}
\frac{\partial(\tilde{f}(\tilde{q})+\tilde{G}(\tilde{q}) \tilde{u})}{\partial \tilde{q}} \frac{\partial \varphi}{\partial q}(q) & =\frac{\partial(\tilde{f} \circ \varphi+\tilde{G} \circ \varphi \tilde{u})}{\partial q} \\
& =\frac{\partial}{\partial q}\left(\frac{\partial \varphi}{\partial q}(f+G \alpha+G \beta \tilde{u})\right) \\
& =\frac{\partial}{\partial q}\left(\frac{\partial \varphi}{\partial q}(f+G u)\right) \\
& =\frac{\partial}{\partial q}\left(\frac{\partial \varphi}{\partial q}\right)(f+G u) \\
& +\frac{\partial \varphi}{\partial q} \frac{\partial(f+G u)}{\partial q} .
\end{aligned}
$$

Using the above expression and the relation

$p^{T}=\tilde{p}^{T} \frac{\partial \varphi}{\partial q}(q)$

we conclude from the second equation of Eq. 16 that

$$
\begin{aligned}
& 0=\dot{p}^{T}+p^{T} \frac{\partial(f+G u)}{\partial q} \\
& =\dot{\tilde{p}}^{T} \frac{\partial \varphi}{\partial q}(q)+\tilde{p}^{T} \frac{\widehat{\partial \varphi}}{\partial q}(q)+\tilde{p}^{T} \frac{\partial \varphi}{\partial q}(q) \frac{\partial(f+G u)}{\partial q} \\
& =\dot{\tilde{p}}^{T} \frac{\partial \varphi}{\partial q}(q) \\
& +\tilde{p}^{T}\left(\frac{\partial}{\partial q}\left(\frac{\partial \varphi}{\partial q}\right)(f+G u)+\frac{\partial \varphi}{\partial q}(q) \frac{\partial(f+G u)}{\partial q}\right) \\
& =\left(\dot{\tilde{p}}^{T}+\tilde{p}^{T} \frac{\partial(\tilde{f}(\tilde{q})+\tilde{G}(\tilde{q}) \tilde{u})}{\partial \tilde{q}}\right) \frac{\partial \varphi}{\partial q}(q)
\end{aligned}
$$

and thus, by invertibility of $\frac{\partial \varphi}{\partial q}(q)$, the second equation of Eq. 16 implies the second equation of Eq. 19.

Next, we have

$$
\tilde{p}^{T} \tilde{G}=\tilde{p}^{T} \frac{\partial \varphi}{\partial q} G \beta=p^{T} G \beta=0,
$$

and thus the third equation of Eq. 16 (the constraint equation) implies the corresponding equation of Eq. 19. Finally,

$\tilde{p}^{T} \tilde{f}=\tilde{p}^{T} \frac{\partial \varphi}{\partial q}(f+g \alpha)=p^{T}(f+g \alpha)=p^{T} f=$ const.

We conclude that $(\tilde{u}(t), \tilde{q}(t), \tilde{p}(t))$ is, indeed, a singular extremal.

We have just proved that the feedback equivalence preserves configuration singularities, and therefore it provides a convenient tool for a description of these singularities. More specifically, given a system $\Sigma$, one needs to find configuration singularities of a normal form $\tilde{\Sigma}$ of $\Sigma$, and then to transform them by the inverse feedback back to the original system $\Sigma$. Later on we shall illustrate this procedure with an example of a space manipulator.

\section{Space Manipulator}

In this section the concept of equivalence and normal forms will be applied to the description of configuration singularities of a free-floating space manipulator built recently in the Space Research Centre (SRC) of the Polish Academy of Sciences. The SRC space manipulator may serve as a prototype device for interception of various objects, like Space debris [15]. The manipulator is portrayed in Fig. 1, while a scheme in Fig. 2, drawn relative to an inertial coordinate frame $(X, Y)$, shows its coordinates and mechanical parameters. The on-board coordinate frame has been denoted as $\left(X_{b}, Y_{b}\right)$.

The SRC space manipulator is composed of a mobile base (a satellite) and a 2DOF planar on-board manipulator. In order to simulate on Earth the lack of gravity, the manipulator is supported by air bearings that enable it to float horizontally over a granite table. The dynamic and geometric parameters of the SRC manipulator can be found in [16]: masses of the links $m_{1}=4.5 \mathrm{~kg}$ and $m_{2}=1.5 \mathrm{~kg}$, lengths of the links $l_{1}=0.619 \mathrm{~m}$ and $l_{2}=0.6 \mathrm{~m}$, positions of centres of mass on the links $d_{1}=0.313 \mathrm{~m}$ and $d_{2}=0.287 \mathrm{~m}$, the mass and the moment of inertia of the base $M=12.9 \mathrm{~kg}$ and $\mathrm{I}=0.208 \mathrm{~kg} \cdot \mathrm{m}^{2}$. It has been demonstrated that in generalized coordinates $\bar{q}=\left(\bar{x}, \bar{y}, \phi, \theta_{1}, \theta_{2}\right)^{T} \in \mathbb{R}^{2} \times \mathbb{T}^{3}$, where bars refer to barycentric position coordinates (the position of the centre of mass) of the base to be specified later on, and $\mathbb{T}^{3}=S^{1} \times S^{1} \times S^{1}$ denotes the 3-dimensional torus, the Lagrangian of the SRC manipulator takes the form

$$
\begin{aligned}
L(\bar{q}, \dot{\bar{q}})= & \frac{1}{2} \mathrm{~A}\left(\dot{\bar{x}}^{2}+\dot{\bar{y}}^{2}\right)+\frac{1}{2} \mathrm{I} \dot{\phi}^{2}+\frac{1}{2} \mathrm{~B}\left(\dot{\phi}+\dot{\theta}_{1}\right)^{2} \\
& +\frac{1}{2} \mathrm{C}\left(\dot{\phi}+\dot{\theta}_{12}\right)^{2} \\
& +\mathrm{D} c_{\theta_{2}}\left(\dot{\phi}+\dot{\theta}_{1}\right)\left(\dot{\phi}+\dot{\theta}_{12}\right) .
\end{aligned}
$$

In this Lagrangian, $m_{12}=m_{1}+m_{2}, \theta_{12}=\theta_{1}+\theta_{2}$, with $s_{\alpha}, c_{\alpha}$ denoting, respectively, $\sin \alpha$ and $\cos \alpha$. Furthermore,

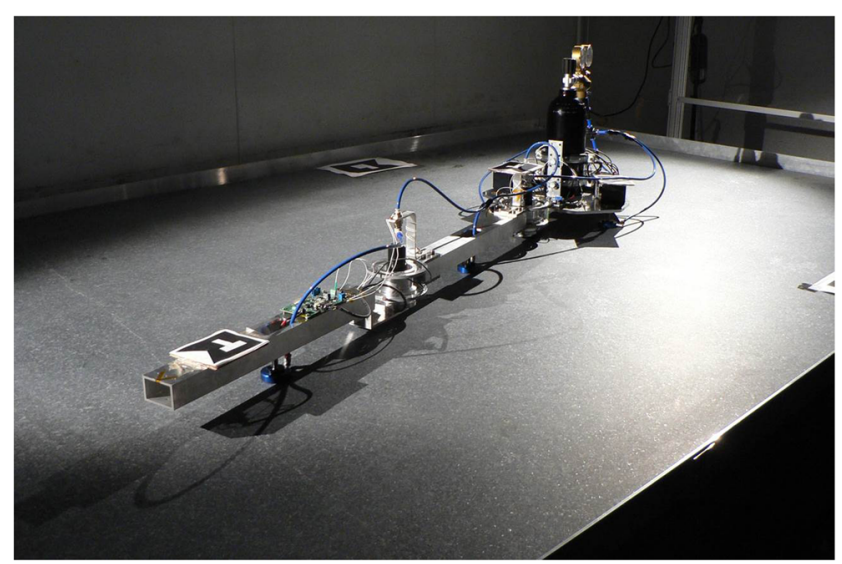

Fig. 1 SRC space manipulator 


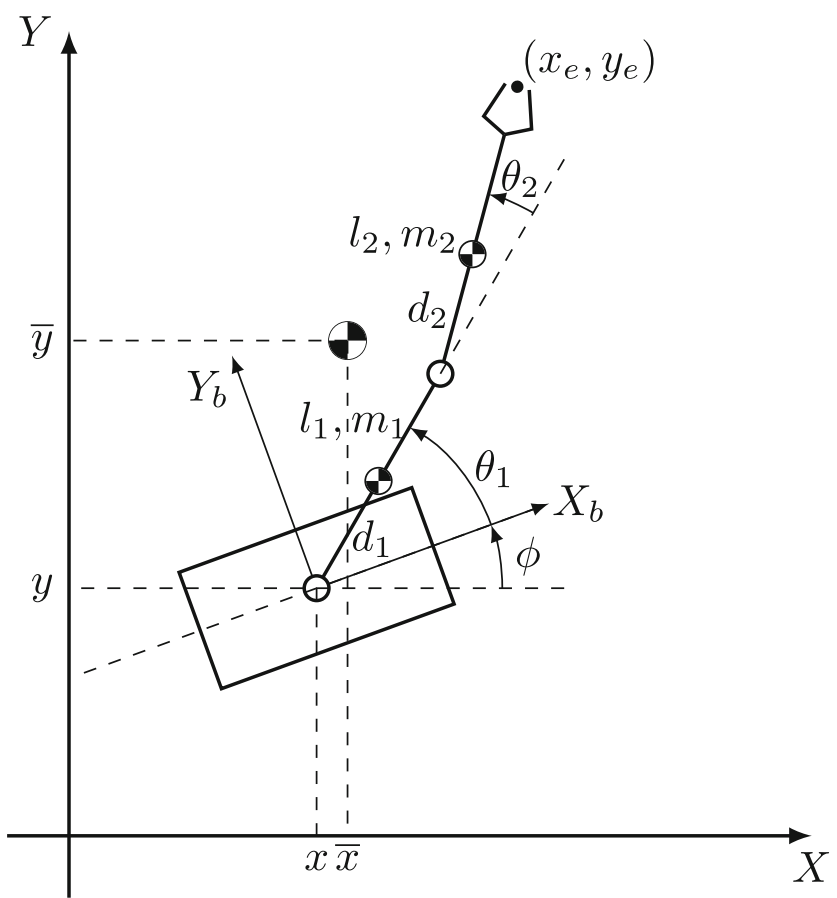

Fig. 2 SRC manipulator: scheme

$\mathrm{A}=M+m_{12}, \mathrm{~B}=\frac{m_{1} m_{2}\left(l_{1}-d_{1}\right)^{2}+M\left(m_{1} d_{1}^{2}+m_{2} l_{1}^{2}\right)}{M+m_{12}}, \mathrm{C}=$ $\frac{\left(M+m_{1}\right) m_{2} d_{2}^{2}}{M+m_{12}}, \mathrm{D}=\frac{m_{1} m_{2}\left(l_{1}-d_{1}\right) d_{2}+M m_{2} l_{1} d_{2}}{M+m_{12}}$. The symbols $\bar{x}$ and $\bar{y}$ denote the barycentric coordinates defined as

$$
\begin{aligned}
\bar{x}= & \frac{1}{M+m_{12}} \times\left(M x+m_{1}\left(x+d_{1} c_{\phi+\theta_{1}}\right)\right. \\
& \left.+m_{2}\left(x+l_{1} c_{\phi+\theta_{1}}+d_{2} c_{\phi+\theta_{12}}\right)\right)
\end{aligned}
$$

and

$$
\begin{aligned}
\bar{y}= & \frac{1}{M+m_{12}} \times\left(M y+m_{1}\left(y+d_{1} s_{\phi+\theta_{1}}\right)\right. \\
& \left.+m_{2}\left(y+l_{1} s_{\phi+\theta_{1}}+d_{2} s_{\phi+\theta_{12}}\right)\right) .
\end{aligned}
$$

The inertia matrix corresponding to the Lagrangian (20) is the following

$Q(\bar{q})=\left[\begin{array}{ccccc}A & 0 & 0 & 0 & 0 \\ 0 & A & 0 & 0 & 0 \\ 0 & 0 & F\left(\theta_{2}\right) & G\left(\theta_{2}\right) & H\left(\theta_{2}\right) \\ 0 & 0 & G\left(\theta_{2}\right) & G\left(\theta_{2}\right) & H\left(\theta_{2}\right) \\ 0 & 0 & H\left(\theta_{2}\right) & H\left(\theta_{2}\right) & H\left(\theta_{2}\right)\end{array}\right]$,

where $F\left(\theta_{2}\right)=\mathrm{I}+\mathrm{B}+\mathrm{C}+2 \mathrm{D} c_{\theta_{2}}, G\left(\theta_{2}\right)=\mathrm{B}+\mathrm{C}+2 \mathrm{D} c_{\theta_{2}}$, $H\left(\theta_{2}\right)=\mathrm{C}+\mathrm{D} c_{\theta_{2}}$. It is easily checked that in the EulerLagrange equations for this Lagrangian both the linear and the angular momenta are conserved. We denote the constant values of the linear momenta by $p_{1}$ and $p_{2}$, and that of the angular momentum by $p_{3}$. The constants $p_{1}, p_{2}, p_{3}$ can be either zero or non-zero. Specifically, we get that the center of mass of the manipulator will move uniformly along a straight line,

$\mathrm{A} \dot{\bar{x}}=\mathrm{p}_{1}, \quad \mathrm{~A} \dot{\bar{y}}=\mathrm{p}_{2}$.

Having described the translational motion of the robot, in the sequel we shall concentrate on its rotational motion. The conservation of the angular momentum results in

$F\left(\theta_{2}\right) \dot{\phi}+G\left(\theta_{2}\right) \dot{\theta}_{1}+H\left(\theta_{2}\right) \dot{\theta}_{2}=\mathrm{p}_{3}$.

We introduce the notation $q=\left(\theta_{1}, \theta_{2}, \phi\right)^{T} \in \mathbb{T}^{3}$. Then, the conservation law (21) takes either the affine Pfaffian form $\mathcal{A}(q) \dot{q}=\mathrm{p}_{3}$, when $\mathrm{p}_{3} \neq 0$, or the linear Pfaffian form $\mathcal{A}(q) \dot{q}=0$, if $\mathrm{p}_{3}=0$.

Introduce the angles

$\varphi=\phi+\theta_{1}, \quad \psi=\phi+\theta_{1}+\theta_{2}$.

The state space of the system is the 3-dimensional torus $\mathbb{T}^{3}=S^{1} \times S^{1} \times S^{1}$, and the original variables $\left(\phi, \theta_{1}, \theta_{2}\right)$ define angle coordinates on $\mathbb{T}^{3} \cong \mathcal{C}=[0,2 \pi] \times[0,2 \pi] \times$ $[0,2 \pi]$, where in the cube $\mathcal{C}$ the values 0 and $2 \pi$ of any of the angles $\phi, \theta_{1}$, and $\theta_{2}$ are identified. Notice that the variables $(\phi, \varphi, \psi)$ are also angle coordinates on $\mathbb{T}^{3}$. Indeed, the cube $\mathcal{C}$ is represented by the parallelepiped

$\mathcal{P}=\left\{(\varphi, \psi, \phi) \in \mathbb{R}^{3}: 0 \leq v_{i} \leq 2 \pi, i=1,2,3\right\}$,

where

$\nu_{1}=\varphi-\phi, \quad v_{2}=\psi-\varphi, \quad v_{3}=\phi$,

and we identify the opposite faces of $\mathcal{P}$ by identifying those points on them that lie on any line parallel to one of the edges. The latter means that for any permutation $(i, j, k)=\sigma(1,2,3)$ and any fixed values $0 \leq c_{i}, c_{j} \leq 2 \pi$ we identify the point satisfying $v_{i}=c_{i}, v_{j}=c_{j}, v_{k}=0$ with that satisfying $v_{i}=c_{i}, v_{j}=c_{j}, v_{k}=2 \pi$. Notice that the variables $(\varphi, \psi, \phi)$ define angle coordinates because, first, for any triplet $\left(d_{1}, d_{2}, d_{3}\right) \in \mathbb{R}^{3}$ the set $\{(\varphi, \psi, \phi)=$ $\left.\left(d_{1}, d_{2}, d_{3}\right)\right\}$ either consists of just one point in $\mathcal{P}$ (with the above identification) or is empty and, second, the variables $(\varphi, \psi, \phi)$ and $(\phi+2 k \pi, \varphi+2 l \pi, \psi+2 m \pi)$ coincide due to the above identification. Therefore, instead of the parallelepiped $\mathcal{P}$, we can consider the cube $\{(\varphi, \psi, \phi)\}=$ $\mathcal{C}^{\prime}=[0,2 \pi] \times[0,2 \pi] \times[0,2 \pi]$ with the values 0 and $2 \pi$ of any of the angles $\phi, \varphi, \psi$ identified.

Next, define the variables

$\alpha=\psi-\varphi, \quad \beta=\psi+\varphi, \quad \gamma=\mathrm{I} \phi+\mathrm{B} \varphi+\mathrm{C} \psi$.

Now observe that introducing the variables $(\alpha, \beta, \gamma)$ maps the cube $\mathcal{C}^{\prime}$ into the parallelepiped

$\mathcal{P}^{\prime}=\left\{(\alpha, \beta, \gamma) \in \mathbb{R}^{3}: 0 \leq \mu_{i} \leq 2 \pi, i=1,2,3\right\}$, 
where

$$
\begin{aligned}
& \mu_{1}=\frac{\beta-\alpha}{2}, \mu_{2}=\frac{\alpha+\beta}{2}, \\
& \mu_{3}=\frac{1}{I}\left(\gamma-\mathrm{B} \frac{\alpha+\beta}{2}-\mathrm{C} \frac{\beta-\alpha}{2}\right),
\end{aligned}
$$

and we identify the opposite faces of $\mathcal{P}^{\prime}$ by identifying those points on them that lie on any line parallel to one of the edges. The latter means (like above) that for any permutation $(i, j, k)=\sigma(1,2,3)$ and any fixed values $0 \leq c_{i}, c_{j} \leq$ $2 \pi$ we identify the point satisfying $\mu_{i}=c_{i}, \mu_{j}=c_{j}$, $\mu_{k}=0$ with that satisfying $\mu_{i}=c_{i}, \mu_{j}=c_{j}, \mu_{k}=2 \pi$. The variables $(\alpha, \beta, \gamma)$ parametrize the parallelepiped $P^{\prime}$, because for any triplet $\left(d_{1}, d_{2}, d_{3}\right) \in \mathbb{R}^{3}$ the set $\{(\alpha, \beta, \gamma)=$ $\left.\left(d_{1}, d_{2}, d_{3}\right)\right\}$ either consists of just one point in $\mathcal{P}^{\prime}$ (with the above identification) or is empty. However, the triplet $(\alpha, \beta, \gamma)$ does not form, in general, angle coordinates on $P^{\prime} \cong \mathbb{T}^{3}$ because the variable $\alpha$ is not periodic (unless a particular rational relation between I, B, and C holds). Notice that already $(\psi+\varphi, \psi-\varphi)$ has to be taken modulo $\pi$ (and not $2 \pi$ ), otherwise any point of $\mathbb{T}^{2}=S^{1} \times S^{1}$ has two different representations as $(\psi+\varphi, \psi-\varphi)$ but the true problem is that the variable $\gamma$ is, in general, not periodic and thus cannot be interpreted as an angle.

Describing a point $q \in \mathbb{T}^{3}$ either as $q=\left(\theta_{1}, \theta_{2}, \phi\right)$ or as $(\varphi, \psi, \phi)$ or as $q=(\alpha, \beta, \gamma)$, the conservation law takes, respectively, the form (21) or one of the two following forms

$\mathrm{I} \dot{\phi}+\mathrm{B} \dot{\varphi}+\mathrm{C} \dot{\psi}+\mathrm{D}(\dot{\varphi}+\dot{\psi}) \cos (\psi-\varphi)=\mathrm{p}_{3}$,

$\dot{\gamma}+\mathrm{D} \dot{\beta} \cos \alpha=\mathrm{p}_{3}$.

For each of them we obtain a global description of the Pfaffian equation as an associated control system. Using $\left(\theta_{1}\right.$, $\left.\theta_{2}, \phi\right)$ as coordinates and choosing $\dot{\theta_{1}}=s_{1}$ and $\dot{\theta_{2}}=s_{2}$ as controls, we get

$\left\{\begin{array}{l}\dot{\theta}_{1}=s_{1} \\ \dot{\theta}_{2}=s_{2} \\ \dot{\phi}=a\left(\theta_{2}\right)+b\left(\theta_{2}\right) s_{1}+c\left(\theta_{2}\right) s_{2},\end{array}\right.$

where $\left(\theta_{1}, \theta_{2}, \phi\right) \in \mathbb{T}^{3}$ and,

$a\left(\theta_{2}\right)=\frac{\mathrm{p}_{3}}{F\left(\theta_{2}\right)}, b\left(\theta_{2}\right)=-\frac{G\left(\theta_{2}\right)}{F\left(\theta_{2}\right)}, c\left(\theta_{2}\right)=-\frac{H\left(\theta_{2}\right)}{F\left(\theta_{2}\right)}$,

with $F\left(\theta_{2}\right)$, by definition, always positive.

Using $(\varphi, \psi, \phi)$ as coordinates and choosing $\dot{\varphi}=w_{1}$ and $\dot{\psi}=w_{2}$ as controls, we obtain

$$
\left\{\begin{array}{l}
\dot{\varphi}=w_{1} \\
\dot{\psi}=w_{2} \\
I \dot{\phi}=\mathrm{p}_{3}-\mathrm{B} w_{1}-\mathrm{C} w_{2}-\left(w_{1}+w_{2}\right) \mathrm{D} \cos (\psi-\varphi),
\end{array}\right.
$$

where $(\varphi, \psi, \phi) \in \mathcal{P}$ (or, equivalently, on $\mathbb{T}^{3}$ ), and $w=$ $\left(w_{1}, w_{2}\right) \in \mathbb{R}^{2}$.
Finally, using $(\alpha, \beta, \gamma)$ as coordinates and choosing as controls $\dot{\alpha}=u_{1}$ and $\dot{\beta}=u_{2}$, we get

$\left\{\begin{array}{l}\dot{\alpha}=u_{1} \\ \dot{\beta}=u_{2} \\ \dot{\gamma}=\mathrm{p}_{3}-u_{2} \mathrm{D} \cos \alpha\end{array}\right.$

where $(\alpha, \beta, \gamma) \in \mathcal{P}^{\prime}, u=\left(u_{1}, u_{2}\right) \in \mathbb{R}^{2}$.

Each of the above representations (27), (29), (30) is global, covers both cases $p_{3}=0$ and $p_{3} \neq 0$, and has its advantages. The first form (27) uses the relative angle coordinates only, while the second form (29) employs the absolute angles, but both are more complicated than the third one. The third form (30) is, indeed, the simplest (there is one nonlinearity only and less terms present) but the price to pay is that we use a coordinate that is not an angle. Moreover, the third form is natural in the sense that the system (for the case $p_{3} \neq 0$ as well for $p_{3}=0$ ) exhibits two different local behaviours (see theorem below), and we need to describe them with the help of a periodic function. The function $\cos \alpha$ encodes those two different behaviours of the system: around its extrema (maxima and minima) and around points of its monotonicity.

By invoking the periodicity of the drift vector field one can show that system (27) (equivalently, (29) and (30)) is controllable, and that its control distribution $\mathcal{G}$ has the growth vector either $(2,3)$, if $\sin \theta_{2} \neq 0$, or $(2,2,3)$ otherwise. The points $q \in \mathbb{T}^{3}$ at which $\sin \theta_{2}=0$ will be referred to as control distribution singularities. We have already noticed that these singularities coincide with the dynamic singularities of the SRC manipulator as well as with the kinematic singularities of the on-board manipulator. This singularity is essential for local normal forms that will be analyzed in the next section.

\subsection{Normal Forms}

The following result establishes the feedback equivalence of our system to some simple polynomial normal forms. Below, by the control system $\Sigma$ we mean the SRC manipulator (for the control-affine case $p_{3} \neq 0$ as well as for the driftless case $p_{3}=0$ ) in one of the feedback equivalent forms (27), or (29), or (30). In those forms, the angle $\theta_{2}$, crucial for the normal forms below, is, respectively, $\theta_{2}=$ $\psi-\varphi=\alpha$.

\section{Theorem 1 Consider the SRC manipulator $\Sigma$.}

1. Suppose that $\mathrm{p}_{3} \neq 0$. Around any $q \in \mathbb{T}^{3}$, such that $\theta_{2} \neq 0, \pi$ (i.e., outside control distribution singularities) the control-affine system $\Sigma$ is locally feedback equivalent to the normal form

$\dot{z}_{1}=v_{1}, \dot{z}_{2}=v_{2}, \dot{z}_{3}=1+z_{1} v_{2}$. 
2. Suppose that $\mathrm{p}_{3} \neq 0$. Around any $q \in \mathbb{T}^{3}$, such that $\theta_{2}=0, \pi$ (i.e., in a neighbourhood of a control distribution singularity) the control-affine system $\Sigma$ is locally feedback equivalent to the normal form

$\dot{z}_{1}=v_{1}, \dot{z}_{2}=v_{2}, \dot{z}_{3}=1+z_{1}^{2} v_{2}$.

3. Suppose that $\mathrm{p}_{3}=0$. Around any $q \in \mathbb{T}^{3}$, such that $\theta_{2} \neq 0$, $\pi$ (i.e., outside control distribution singularities) the driftless system $\Sigma$ is locally feedback equivalent to the normal form

$\dot{z}_{1}=v_{1}, \dot{z}_{2}=v_{2}, \dot{z}_{3}=z_{1} v_{2}$.

4. Suppose that $\mathrm{p}_{3}=0$. Around any $q \in \mathbb{T}^{3}$, such that $\theta_{2}=0, \pi$ (i.e., in a neighbourhood of a control distribution singularity) the driftless system $\Sigma$ is locally feedback equivalent to the normal form

$\dot{z}_{1}=v_{1}, \dot{z}_{2}=v_{2}, \dot{z}_{3}=z_{1}^{2} v_{2}$.

The proof of the above theorem reduces to two propositions below, where we provide explicit transformations bringing Eq. 27 into Eq. 29 and the latter into Eq. 30, given in Proposition 2, and those bringing Eq. 30 into the four normal forms of Theorem 1, given in Proposition 3. In the two propositions we shall also describe the regions, where the system admits the above normal forms.

Notice that there are only four possible local behaviours of the SRC manipulator: two for $p_{3} \neq 0$ and two for $p_{3}=0$. What matters is to distinguish the extrema of $\cos \theta_{2}$ from the regions of its monotonicity (neither matters whether the extremum is a minimum or a maximum nor whether $\cos \theta_{2}$ is growing up or descending). Moreover, the normal forms do not depend on the actual values of $p_{3} \neq 0$ neither do on the values of the mechanical parameters I, B, C, D. Therefore the local behaviour of the system does not depend on them either.

A feedback classification of typical control-affine systems with 3 states and 2 inputs is given in [7] (see also [17] for systems in dimension $n$ ). For a system of the form

$\dot{q}=f(q)+g_{1}(q) u_{1}+g_{2}(q) u_{2}$,

on a 3-dimensional configuration space $Q$, the crucial objects, as argued in [7], are two sets

$$
\begin{aligned}
E(q) & =\left\{q \in Q: \operatorname{det}\left(f, g_{1}, g_{2}\right)(q)=0\right\} \\
S(q) & =\left\{q \in Q: \operatorname{det}\left(g_{1}, g_{2},\left[g_{1}, g_{2}\right]\right)(q)=0\right\},
\end{aligned}
$$

where $\left[g_{1}, g_{2}\right]$ is the Lie bracket of the vector fields $g_{1}$ and $g_{2}$. The equilibrium set $E$ consists of points $q$ that can become an equilibrium of $f$ via a feedback. The set $S$ consists of singularities of the control distribution $\mathcal{G}$. Using $E$ and $S$, a geometric description of our four normal forms for SRC becomes very transparent. If $p_{3} \neq 0$, then the equilibrium set $E$ of the SRC manipulator (27) (equivalently, of Eq. 29 and of Eq. 30) is empty since we cannot immobilize the manipulator if the angular momentum $p_{3}$ is nonzero. We thus get two normal forms (31) and (32) depending on whether the nominal point satisfies, respectively, $q \notin S$ or $q \in S$ (equivalently, $\theta_{2} \neq 0, \pi$ or $\theta_{2}=0, \pi$ ). On the other hand, if $\mathrm{p}_{3}=0$, then $E=\mathbb{T}^{3}$ and the normals forms (33) and (34) are distinguished by the nominal point that satisfies $q \notin S$ or $q \in S$ for, respectively, (33) or (34) (like in the case $p_{3} \neq 0$ ).

To compare our results with those of [7], recall that in the latter it is proved that outside the union $E \cup S$, any system is locally feedback equivalent to the normal form (31). As we have just observed, if $p_{3} \neq 0$ then for the SRC manipulator (27) the equilibrium set is always empty, so outside the singularities of $\mathcal{G}$ we can transform it to the form (31). Therefore, item 1. of Theorem 1 is, actually, a consequence of [7]. At points $q \in S$ and outside $E$, the authors of [7] propose also a normal form under two genericity assumption. The first is that at $q \in S$, the vector fields $g_{1}, g_{2}$, and $h$ are independent, where $h$ is either $\left[g_{1},\left[g_{1}, g_{2}\right]\right]$ or $\left[g_{2},\left[g_{1}, g_{2}\right]\right]$. This assumption implies that $S$ is a surface in $Q$ and it is satisfied for the SRC manipulator: $S$ is actually the union of two surfaces (two 2-dimensional tori) $\left\{\theta_{2}=0\right\}$ and $\left\{\theta_{2}=\right.$ $\pi\}$. The second genericity assumption of [7] is that for one (and thus any) vector field $g \in \mathcal{G}$ satisfying $g(q) \in T_{q} S$ for any $q \in S$, we should have $g$ and $[f, g]$ independent at $q \in S$. This assumption is not satisfied for the SRC manipulator because the linear approximation of system (27) (equivalently, of Eq. 29 and of Eq. 30) along any trajectory contained in $S$ is not controllable (while the condition of [7] requires that it should be). Item 2. of Theorem 1 gives a normal form in that case, which is a new result.

In the case of the space manipulator with zero angular momentum, $\mathrm{p}_{3}=0$, system (27) (equivalently, (29) and (30)) becomes driftless, and its normal forms (33) and (34) under feedback equivalence (consisting of a change of coordinates $z=\varphi(q)$ and a linear transformations of controls $u=\beta(q) v)$ are, actually, corollaries of the calssical Darboux and Martinet normal forms for distributions; see, e.g. [18].

Now we shall give transformations bringing (27) into the above normal forms in both the control-affine and the driftless cases. The existence of transformations yielding (31) is proved in [7] via a homotopy method that is less constructive, so also for that case we shall give our construction that provides explicit formulae. We shall separate the description of transformations mapping (27) into (29) and the latter into (30), given in Proposition 2, from the description of those bringing (30) into the four normal forms of Theorem 1, given in Proposition 3. 
Proposition 2 Consider the SRC manipulator in both cases $\mathrm{p}_{3} \neq 0$ and $\mathrm{p}_{3}=0$.

1. The global angle transformation

$$
\left[\begin{array}{l}
\theta_{1} \\
\theta_{2} \\
\phi
\end{array}\right] \mapsto\left[\begin{array}{c}
\varphi \\
\psi \\
\phi
\end{array}\right]=\left[\begin{array}{c}
\phi+\theta_{1} \\
\phi+\theta_{1}+\theta_{2} \\
\phi
\end{array}\right]
$$

maps $\mathbb{T}^{3}$ onto $\mathbb{T}^{3}$ and followed by the invertible static feedback

$$
\left[\begin{array}{l}
w_{1} \\
w_{2}
\end{array}\right]=\frac{1}{F}\left[\begin{array}{l}
\mathrm{p}_{3} \\
\mathrm{p}_{3}
\end{array}\right]+\frac{1}{F}\left[\begin{array}{l}
F-G-H \\
F-G
\end{array}\right]\left[\begin{array}{l}
s_{1} \\
s_{2}
\end{array}\right]
$$

brings system (27) into Eq. 29.

2. The global transformation

$$
\left[\begin{array}{l}
\varphi \\
\psi \\
\phi
\end{array}\right] \mapsto\left[\begin{array}{l}
\alpha \\
\beta \\
\gamma
\end{array}\right]=\left[\begin{array}{l}
\psi-\varphi \\
\psi+\varphi \\
\mathrm{I} \phi+\mathrm{B} \varphi+\mathrm{C} \psi
\end{array}\right]
$$

maps $\mathbb{T}^{3}$ onto $\mathcal{P}^{\prime}$ and followed by the invertible static feedback

$$
\left[\begin{array}{l}
u_{1} \\
u_{2}
\end{array}\right]=\left[\begin{array}{ll}
-1 & 1 \\
1 & 1
\end{array}\right]\left[\begin{array}{l}
w_{1} \\
w_{2}
\end{array}\right]
$$

brings system (29) into Eq. 30.

Proof By a direct calculation we check that, indeed, the transformations proposed in item 1. and 2. are global maps between the indicated state spaces and bring, respectively, (27) into (29) and (29) into (30).

Below we shall describe transformations bringing (30) into the normal forms of Theorem 1. Obviously, normalizing system (27) or (29) will require applying, respectively, preliminary transformations (37)-(38) or (39)-(40) described above. Recall that system (30) is considered on the parallelepiped $\mathcal{P}^{\prime}$.

Proposition 3 Assume $\mathrm{p}_{3} \neq 0$.

1. If $\alpha_{0}=\theta_{20} \neq 0, \pi$, then the transformation

$$
\left[\begin{array}{l}
\alpha \\
\beta \\
\gamma
\end{array}\right] \mapsto\left[\begin{array}{l}
z_{1} \\
z_{2} \\
z_{3}
\end{array}\right]=\left[\begin{array}{c}
\cos \alpha \\
-\frac{\mathrm{D}}{\mathrm{p}_{3}} \beta \\
\frac{1}{\mathrm{p}_{3}} \gamma
\end{array}\right]
$$

followed by the invertible static feedback

$$
\left[\begin{array}{l}
v_{1} \\
v_{2}
\end{array}\right]=\left[\begin{array}{cc}
-\sin \alpha & 0 \\
0 & -\frac{\mathrm{D}}{\mathrm{p}_{3}}
\end{array}\right]\left[\begin{array}{l}
u_{1} \\
u_{2}
\end{array}\right]
$$

transforms system (30), considered on $\mathcal{P}^{\prime} \cap\{0<\alpha<$ $\pi\}$ if $0<\theta_{20}<\pi$, or on $\mathcal{P}^{\prime} \cap\{\pi<\alpha<2 \pi\}$ if $\pi<\theta_{20}<2 \pi$, into the normal form (31).
2. If $\alpha_{0}=\theta_{20}=0, \pi$, then the transformation

$$
\left[\begin{array}{l}
\alpha \\
\beta \\
\gamma
\end{array}\right] \mapsto\left[\begin{array}{l}
z_{1} \\
z_{2} \\
z_{3}
\end{array}\right]=\left[\begin{array}{c}
(1 \mp \cos \alpha)^{1 / 2} \\
\pm \frac{\mathrm{D}}{\mathrm{p}_{3}} \beta \\
\frac{1}{\mathrm{p}_{3}}(\gamma \pm \mathrm{D} \beta)
\end{array}\right]
$$

followed by the invertible static feedback

$\left[\begin{array}{l}v_{1} \\ v_{2}\end{array}\right]=\left[\begin{array}{cc}\frac{ \pm \sin \alpha}{2(1 \mp \cos \alpha)^{1 / 2}} & 0 \\ 0 & \pm \frac{\mathrm{D}}{\mathrm{p}_{3}}\end{array}\right]\left[\begin{array}{l}u_{1} \\ u_{2}\end{array}\right]$,

where in the symbols “ \pm " and “干” the upper sign corresponds to $\theta_{20}=0$ and the lower sign to $\theta_{20}=\pi$, transforms system (30), considered on $\mathcal{P}^{\prime} \cap\left\{-\frac{\pi}{2}<\alpha<\right.$ $\left.\frac{\pi}{2}\right\}$ if $-\frac{\pi}{2}<\theta_{20}<\frac{\pi}{2}$, or on $\mathcal{P}^{\prime} \cap\left\{\frac{\pi}{2}<\alpha<\frac{3 \pi}{2}\right\}$ if $\frac{\pi}{2}<\theta_{20}<\frac{3 \pi}{2}$ into the normal form (32).

If $\mathrm{p}_{3}=0$, then the above transformations, with $\mathrm{p}_{3}$ replaced by one, bring the driftless system (30), in which $\mathrm{p}_{3}=0$, into, respectively, the normal forms (33) and (34).

Remark 1 The transformation of item 1. maps $\theta_{20}$ in the two considered cases of its placement into, respectively, $z_{10}=\frac{\pi}{2}$ and $z_{10}=\frac{3 \pi}{2}$ while the transformation of item 2. maps $\theta_{20}$ in the two considered cases into, respectively, $z_{10}=0$ and $z_{10}=\pi$.

Proof By a direct calculation we check that, indeed, the transformations proposed in item 1. and 2. map, respectively Eq. 30 into Eqs. 31 and 30 into Eq. 32.

Because it is customary in robotics to describe joint rotations by the relative angles, it might be interesting to go directly from the original system (27) into its normal forms. Combining the above propositions we get:

Corollary 1 Assume $\mathrm{p}_{3} \neq 0$.

1. If $\theta_{20} \neq 0, \pi$, then the transformation

$$
\begin{aligned}
{\left[\begin{array}{l}
\theta_{1} \\
\theta_{2} \\
\phi
\end{array}\right] } & \mapsto\left[\begin{array}{l}
z_{1} \\
z_{2} \\
z_{3}
\end{array}\right] \\
= & {\left[\begin{array}{c}
\cos \theta_{2} \\
-\frac{\mathrm{D}}{\mathrm{p}_{3}}\left(2 \phi+2 \theta_{1}+\theta_{2}\right) \\
\frac{1}{\mathrm{p}_{3}}\left((\mathrm{I}+\mathrm{B}+\mathrm{C}) \phi+(\mathrm{B}+\mathrm{C}) \theta_{1}+\mathrm{C} \theta_{2}\right)
\end{array}\right] }
\end{aligned}
$$

followed by the invertible static feedback

$$
\left[\begin{array}{l}
v_{1} \\
v_{2}
\end{array}\right]=\left[\begin{array}{l}
0 \\
-\frac{2 a \mathrm{D}}{\mathrm{p}_{3}}
\end{array}\right]+\left[\begin{array}{cc}
0 & -\sin \theta_{2} \\
-\frac{2 \mathrm{D}}{\mathrm{p}_{3}}(b+1) & -\frac{2 \mathrm{D}}{\mathrm{p}_{3}}\left(c+\frac{1}{2}\right)
\end{array}\right]\left[\begin{array}{l}
s_{1} \\
s_{2}
\end{array}\right],
$$

with $a, b$ and $c$ given by (28), transforms system (27) into the normal form (31). 
2. If $\alpha_{0}=\theta_{20}=0, \pi$, then the transformation

$$
\left[\begin{array}{c}
\theta_{1} \\
\theta_{2} \\
\phi
\end{array}\right] \mapsto\left[\begin{array}{c}
z_{1} \\
z_{2} \\
z_{3}
\end{array}\right]=\left[\begin{array}{c}
\operatorname{sgn} \theta_{2}\left(1 \mp \cos \theta_{2}\right)^{1 / 2} \\
\pm \frac{\mathrm{D}}{\mathrm{p}_{3}}\left(2 \phi+2 \theta_{1}+\theta_{2}\right) \\
\frac{1}{\mathrm{p}_{3}}\left((\mathrm{I}+\mathrm{B}+\mathrm{C} \pm 2 \mathrm{D}) \phi+(\mathrm{B}+\mathrm{C} \pm 2 \mathrm{D}) \theta_{1}+(\mathrm{C} \pm \mathrm{D}) \theta_{2}\right)
\end{array}\right]
$$

followed by the invertible static feedback

$$
\begin{aligned}
{\left[\begin{array}{l}
v_{1} \\
v_{2}
\end{array}\right]=} & {\left[\begin{array}{l}
0 \\
\pm \frac{2 a \mathrm{D}}{\mathrm{p}_{3}}
\end{array}\right] } \\
& +\left[\begin{array}{cc}
0 & \pm \operatorname{sgn} \theta_{2} \frac{\sin \theta_{2}}{2\left(1 \mp \cos \theta_{2}\right)^{1 / 2}} \\
\pm \frac{2 \mathrm{D}}{\mathrm{p}_{3}}(b+1) & \pm \frac{2 \mathrm{D}}{\mathrm{p}_{3}}\left(c+\frac{1}{2}\right)
\end{array}\right]\left[\begin{array}{l}
s_{1} \\
s_{2}
\end{array}\right],
\end{aligned}
$$

where in the symbols “ \pm " and “ $\mp$ ” the upper sign corresponds to $\theta_{20}=0$ and the lower sign to $\theta_{20}=\pi$, transforms system (27) into the normal form (32).

If $\mathrm{p}_{3}=0$, then the above transformations, with $\mathrm{p}_{3}$ replaced by one, bring the driftless system (27), in which $\mathrm{p}_{3}=0$, into, respectively, the normal forms (33) and (34).

All these results on normal forms are summarized in the following diagrams. For $p_{3} \neq 0$ :

\begin{tabular}{|c|c|}
\hline \multicolumn{2}{|c|}{$\operatorname{system}(27), \mathrm{p}_{3} \neq 0$} \\
\hline$\theta_{2} \neq 0, \pi$ & $\theta_{2}=0 \vee \pi$ \\
\hline$\swarrow$ & $\searrow$
\end{tabular}

feedback (45), (46)

$\downarrow$

normal form (31)

For $\mathrm{p}_{3}=0$ :

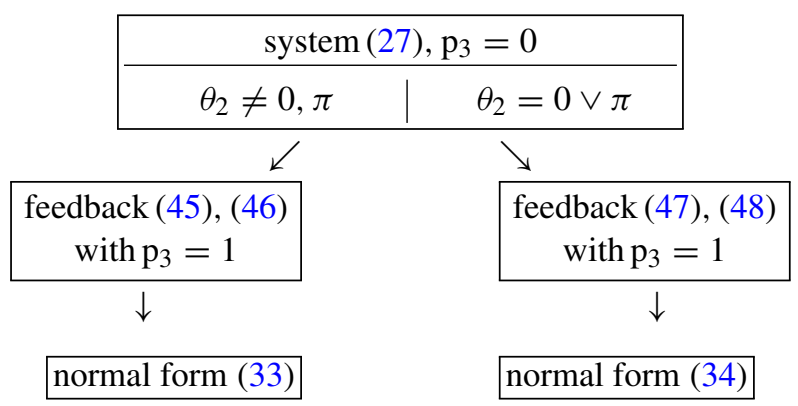

\subsection{Configuration Singularities of the Normal Forms}

In this section we shall describe configuration singularities of the SRC manipulator. We shall do it with the help of the normal forms of Theorem 1, and then we shall provide a global description using the representation (29). Recall that $S=\left\{z_{1}=0\right\}=\left\{\sin \theta_{2}=0\right\}$ is the set (which is, actually, a surface formed by two tori) of singularities of the control distribution $\mathcal{G}$, that consists of points at which the growth vector is $(2,2,3)$, whereas it is $(2,3)$ outside $S$.

\subsubsection{The Case $q \notin S$}

We shall consider the normal forms (31) and (33). The control vector fields are $g_{1}(z)=(1,0,0)^{T}$ and $g_{2}(z)=(0,1$, $\left.z_{1}\right)^{T}$, the drift $f(z)=(0,0, \epsilon)^{T}, \epsilon=0,1$ for, respectively, $\mathrm{p}_{3}=0$ and $\mathrm{p}_{3} \neq 0$. In accordance with (15) a singular extremal $(v(t), z(t), p(t))$ should satisfy the equations

$\dot{z}=f(z)+g_{1}(z) v_{1}+g_{2}(z) v_{2}, \quad \dot{p}^{T}=-p^{T} A, \quad p^{T} B=0$, where $A=\left[\begin{array}{lll}0 & 0 & 0 \\ 0 & 0 & 0 \\ v_{2} & 0 & 0\end{array}\right]$ and $B=\left[\begin{array}{ll}1 & 0 \\ 0 & 1 \\ 0 & z_{1}\end{array}\right]$. Thus, the following identities have to be satisfied

$p_{1}=0, p_{3} z_{1}+p_{2}=0, \dot{p}_{2}=\dot{p}_{3}=0, \dot{p}_{1}=-p_{3} v_{2}$.

In consequence, we get $p_{2}=c_{2}=$ const, $p_{3}=c_{3}=$ const, $p_{3} v_{2}=0$. The last equality implies that either $p_{3}=$ $0, v_{2}$ arbitrary or $p_{3} \neq 0$ and $v_{2}=0$. The former possibility leads to $p_{2}=0$, which is not permitted because of $p \neq 0$, so the only possibility is $p_{3}=c_{3} \neq 0$ and $v_{2}=0$. Further, from $p_{3} z_{1}+p_{2}=0$, it follows that $z_{1}=-\frac{c_{2}}{c_{3}}=$ const, so $v_{1}=\dot{z}_{1}=0$. We have shown that in the case $q \notin S$ the configuration singularities are trivial, namely, they are zero controls. Therefore in the driftless case $p_{3}=0$, singular control-state trajectories are $(v(t), z(t))=(0, z(0))$. and thus singular curves consist of stationary points $\left\{z_{0}=z(0)\right\}$. In the control-affine case $p_{3} \neq 0$, singular control-state trajectories are $(v(t), z(t))=(0, z(t))$, where the singular curves are (in the $z$-coordinates) the straight lines $z(t)=$ $\left(z_{10}, z_{20}, t+z_{30}\right)$.

\subsubsection{The Case $q \in S$}

Now consider the normal forms (32) and (34). Put $\epsilon$ equal 0 or 1 if $\mathrm{p}_{3}$ is, respectively, 0 or non-zero. According to Eq. 15, the singular extremals $(v(t), z(t), p(t))$ should fulfil

$\dot{z}=f(z)+g_{1}(z) v_{1}+g_{2}(z) v_{2}$,

where $f(z)=(0,0, \epsilon)^{T}, g_{1}(z)=(1,0,0)^{T}$ and $g_{2}(z)=$ $\left(0,1, z_{1}^{2}\right)^{T}$, together with the identities

$\dot{p}^{T}=-p^{T} A, p^{T} B=0$, 


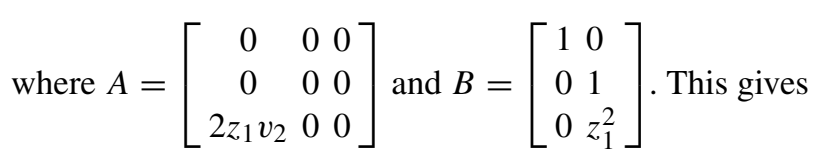
$p_{1}=0, p_{3} z_{1}^{2}+p_{2}=0, \dot{p}_{1}=-2 p_{3} z_{1} v_{2}, \dot{p}_{2}=\dot{p}_{3}=0$.

Like above, we conclude that $p_{2}=c_{2}=$ const, $p_{3}=c_{3}=$ const, and thus $z_{1}^{2}=-\frac{c_{2}}{c_{3}}=$ const, so $v_{1}=\dot{z}_{1}=0$. Since $\dot{p}_{1}=0=-2 p_{3} z_{1} v_{2}$, it follows that either $p_{3}=0$, or $v_{2}=$ 0 , or $z_{1}=0$ The first case implies that $p_{2}=0$, which is impossible (recall that $p \neq 0$ ). The second case $v_{2}=0$ gives singular controls $\left(v_{1}(t), v_{2}(t)\right)=(0,0)$, which is exactly the case described above. Finally, in the third case $z_{1}=0$, we get non-trivial singular controls $\left(v_{1}(t), v_{2}(t)\right)=$ $\left(0, v_{2}(t)\right)$, with $v_{2}(t)$ any control function. This, depending on the value of $p_{3}$, gives the following singular curves. In the driftless case $p_{3}=0$, the surface $S=\left\{z_{1}=0\right\}$ is foliated by the singular curves $z(t)=\left(0, V(t)+z_{20}, z_{30}\right)$, where $V(t)=\int_{0}^{t} v_{2}(s) d s$. In other words, outside $S$, singular curves are stationary points only, while on $S=\left\{z_{1}=0\right\}$, on which $\left(z_{2}, z_{3}\right)$ form coordinates, $z_{3}$ remains unchanged and $z_{2}(t)$ can change arbitrarily. In the control-affine case $\mathrm{p}_{3} \neq$ 0 , outside $S$, the singular curves are straight lines $z(t)=$ $\left(z_{10}, z_{20}, t+z_{30}\right)$ and on the surface $S=\left\{z_{1}=0\right\}$ they are $z(t)=\left(0, V(t)+z_{20}, t+z_{30}\right)$, where $V(t)$ is the same as above. In other words, on $S$, equipped with the coordinates $\left(z_{2}, z_{3}\right)$, they are trajectories of the uncontrollable system $\dot{z}_{2}=v_{2}(t), \dot{z}_{3}=1$.

\subsubsection{Configuration Singularities of the SRC Manipulator}

The above analysis, based on normal forms, gives all configuration singularities. Now, we shall explain what the global description of them is for the SRC manipulator. To this end, we shall use model (29). A singular extremal $(w(t), q(t)$, $p(t))$ should satisfy the equations

$\dot{q}=f(q)+g_{1}(q) w_{1}+g_{2}(q) w_{2}, \quad \dot{p}^{T}=-p^{T} A, \quad p^{T} B=0$, where $q=(\varphi, \psi, \phi)$,

$$
A(t)=\frac{\mathrm{D}}{\mathrm{I}}\left[\begin{array}{ccc}
0 & 0 & 0 \\
0 & 0 & 0 \\
-\left(w_{1}+w_{2}\right) \sin (\psi-\varphi)\left(w_{1}+w_{2}\right) \sin (\psi-\varphi) & 0
\end{array}\right]
$$

and

$$
B(t)=\left[\begin{array}{cc}
1 & 0 \\
0 & 1 \\
-\frac{\mathrm{B}}{\mathrm{I}}-\frac{\mathrm{D}}{\mathrm{I}} \cos (\psi-\varphi) & -\frac{\mathrm{C}}{\mathrm{I}}-\frac{\mathrm{D}}{\mathrm{I}} \cos (\psi-\varphi)
\end{array}\right] .
$$

Thus, the following identities have to be satisfied

$$
\begin{aligned}
& \dot{p}_{1}=p_{3} \frac{\mathrm{D}}{I}\left(w_{1}+w_{2}\right) \sin (\psi-\varphi) \\
& \dot{p}_{2}=-p_{3} \frac{\mathrm{D}}{\mathrm{I}}\left(w_{1}+w_{2}\right) \sin (\psi-\varphi), \\
& p_{3}=c_{3}=\text { const },
\end{aligned}
$$

and

$I p_{1}=p_{3}(\mathrm{~B}+\mathrm{D} \cos (\psi-\varphi)), I p_{2}=p_{3}(\mathrm{C}+\mathrm{D} \cos (\psi-\varphi))$.

It follows that $p_{1}-p_{2}=p_{3} \frac{\mathrm{B}-\mathrm{C}}{\mathrm{I}}$ and hence $\dot{p}_{1}-\dot{p}_{2}=0$. From Eq. 49, we conclude that

$\dot{p}_{1}-\dot{p}_{2}=2 p_{3} \frac{\mathrm{D}}{\mathrm{I}}\left(w_{1}+w_{2}\right) \sin (\psi-\varphi)=0$

and thus either $p_{3}=0$ (implying $p_{1}=p_{2}=0$, which is impossible), or $w_{1}+w_{2}=0$, or $\sin (\psi-\varphi)=0$. If $w_{1}+w_{2}=0$, then $\dot{p}_{1}=0$, hence $p_{1}=c_{1}=$ const and $p_{2}=c_{2}=$ const . Therefore $\cos (\psi-\varphi)=$ const implying that $w_{2}-w_{1}=0$. Thus $\left(w_{1}(t), w_{2}(t)\right)=(0,0)$ are singular controls. They lead to the singular curves $(\varphi(t), \psi(t)$, $\phi(t))=\left(\varphi_{0}, \psi_{0}, \frac{\mathrm{p}_{3}}{\mathrm{I}} t+\phi_{0}\right)$. In other words, the angles $\varphi$ and $\psi$ remain constant, and the angle $\phi$ rotates with the constant angular velocity $\frac{\mathrm{p}_{3}}{\mathrm{I}}$.

In the third case $\sin (\psi-\varphi)=0$, we have either $\theta_{2}=$ $\psi-\varphi=0$ or $\theta_{2}=\psi-\varphi=\pi$. The surface of control distribution singularities is, as we have already observed, formed by two 2-dimensional tori $\left\{\theta_{2}=0\right\} \cup\left\{\theta_{2}=\pi\right\}$ and the system on them obeys the equations

$$
\begin{aligned}
& \dot{\varphi}=w_{1} \\
& \dot{\theta}_{2}=0 \\
& \mathrm{I} \dot{\phi}=\mathrm{p}_{3}-(\mathrm{B}+\mathrm{C} \pm 2 \mathrm{D}) w_{1} .
\end{aligned}
$$

The singular controls are $\left(w_{1}(t), w_{2}(t)\right)$, such that the control $w_{1}(t)=w_{2}(t)$ is arbitrary, and the corresponding singular curves are trajectories of the above control system evolving on one of the surfaces $\left\{\theta_{2}=0\right\}$ or $\left\{\theta_{2}=\pi\right\}$ on which $(\varphi, \phi)$ form a system of global angle coordinates. The system

$$
\begin{aligned}
& \dot{\varphi}=w_{1} \\
& \mathrm{I} \dot{\phi}=\mathrm{p}_{3}-(\mathrm{B}+\mathrm{C} \pm 2 \mathrm{D}) w_{1} .
\end{aligned}
$$

is not controllable, and in order to perform its decomposition into controllable/uncontrollable subsystems, we have to introduce the variable

$\delta=\gamma \pm \mathrm{D}(\beta-\alpha)=\mathrm{I} \phi+\mathrm{B} \varphi+\mathrm{C} \psi \pm 2 \mathrm{D} \varphi$,

with the same variables $\alpha, \beta$, and $\gamma$ that have already appeared in Eq. 24 and in the representation (30), yielding the following control systems on $\left\{\theta_{2}=0\right\}$ and $\left\{\theta_{2}=\pi\right\}$

$\dot{\varphi}=w_{1}$
$\dot{\delta}=p_{3}$

Depending on relations (rational or not) between mechanical parameters $\mathrm{p}_{3}, \mathrm{~B}, \mathrm{C}, \mathrm{D}, \mathrm{I}$ of the system, the corresponding singular curve can be either a periodic trajectory or a dense trajectory on one of the tori $\mathbb{T}^{2} \cong\left\{\left(\varphi, \theta_{2}, \phi\right): \varphi \in\right.$ $\left.S^{1}, \phi \in S^{1}, \theta_{2}=0\right\}$ or $\mathbb{T}^{2} \cong\left\{\left(\varphi, \theta_{2}, \phi\right): \varphi \in S^{1}, \phi \in\right.$ $\left.S^{1}, \theta_{2}=\pi\right\}$. 


\subsubsection{Comparing the Driftless and Control-Affine Cases}

Normal forms of Theorem 1 in the driftless and controlaffine case are almost identical (the only difference is the presence of the constant drift vector field $(0,0,1)^{T}$ if $p_{3} \neq$ 0 ). This is a consequence of the fact that the geometry of both cases is similar. Let $z_{t}^{0}(q)$ denote the solution of Eq. 31 passing for $t=0$ through $q$, and corresponding to the zero control $v=0$. Then the derivative $\frac{\partial z_{t}^{0}(q)}{\partial q}$, denoted by $\left(z_{t}^{0}\right)_{*}$, is a symmetry of the control distribution $\mathcal{G}$, that is, maps $\mathcal{G}(q)$ into $\mathcal{G}(p)$, where $p=z_{t}^{0}(q)$. Actually, $\left(z_{t}^{0}\right)_{*}$ maps $g_{1}$ into $g_{1}$ and $g_{2}$ into $g_{2}$. The same is true for the normal form (32). This explains why for our normal forms, the singular curves of the control-affine system are simply the singular curves of the driftless system shifted by translations along the third component being solutions of the uncontrolled system.

The same analysis applies to system (29). Denote $\xi=$ $(\varphi, \psi, \phi)^{T}$ and represent (29) as

$\dot{\xi}=f(\xi)+w_{1} g_{1}(\xi)+w_{2} g_{2}(\xi)$.

Then $\left[f, g_{1}\right]=\left[f, g_{2}\right]=0$ implying that the flows (solutions) of $f$ and $g_{1}$ as well as those of $f$ and $g_{2}$ commute. Denote by $\xi_{t}^{0}(q)$ the solution of Eq. 29, passing by $q$ for $t=0$ and corresponding to $w_{1}=w_{2}=0$, by $\xi_{t}^{a, w}(q)$ the solution corresponding to $w=\left(w_{1}(t), w_{2}(t)\right)$, and by $\xi_{t}^{d, w}(q)$ the solution corresponding to $w=\left(w_{1}(t), w_{2}(t)\right)$ with $f \equiv 0$ (the superscripts $a$ and $d$ referring to, respectively, control-affine and driftless system). Then

$\xi_{t}^{a, w}(q)=\xi_{t}^{0}\left(\xi_{t}^{d, w}(q)\right)=\xi_{t}^{d, w}\left(\xi_{t}^{0}(q)\right)$.

In other words, if we apply to control-affine system (29) a control $w=\left(w_{1}(t), w_{2}(t)\right)$, we may, equivalently, apply $w=\left(w_{1}(t), w_{2}(t)\right)$ to the driftless system followed by putting $w=0$ (or the same in the reversed order). This explains similarities between configuration singularities of the control-affine and the driftless system; this also proves that the derivative $\left(\xi_{t}^{0}\right)_{*}$ is a symmetry of the control distribution $\mathcal{G}$ (actually, of the control vector fields $g_{1}$ and $g_{2}$ ) and, finally, shows that controlling the control-affine system reduces to controlling the driftless one.

Notice that an analogous observation applies to system (30), but not to Eq. 27, because for the latter the Lie bracket of the drift with the control vector fields does not vanish (that is, the solutions of the corresponding differential equations do not commute), and the flow of the drift does not preserve the control distribution.

\subsection{Singular Controllability Gramian}

As has been mentioned, performance of Jacobian motion planning algorithms deteriorates around configuration singularities. In support of this statement we shall show explicitly that the controllability Gramian (7) entering the Jacobian motion planning algorithm (11) based on the Jacobian pseudoinverse (14) gets rank deficient at configuration singularities of the SRC manipulator.

To this objective we first determine configuration singularities and singular curves for system (27) representing the space manipulator's dynamics. Using the characterization of configuration singularities of the normal forms provided in Section 7.2 and the inverse feedback transformations (45) (46) or (47)-(48) we get the following results. Denote $\theta_{10}=$ $\theta_{1}(0), \theta_{20}=\theta_{2}(0)$, and $\phi_{0}=\phi(0)$.

Corollary 21 . If $\sin \theta_{20} \neq 0$ then configuration singularities of Eq. 27 are

$$
s_{1}=-\frac{a\left(\theta_{20}\right)}{b\left(\theta_{20}\right)+1}, \quad s_{2}=0,
$$

and the singular curve

$$
\theta_{1}=\theta_{10}+s_{1} t, \quad \theta_{2}=\theta_{20}, \quad \phi=\phi_{0}-s_{1} t
$$

2. For $\sin \theta_{20}=0$, configuration singularities of Eq. 27 are

$s_{1}=s_{1}(t)$, arbitrary, and $s_{2}=0$,

while the singular curve is

$$
\begin{aligned}
& \theta_{1}=\int_{0}^{t} s(\tau) d \tau+\theta_{10}, \quad \theta_{2}=\theta_{20}=0 \text { or } \pi, \\
& \phi=\phi_{0}+a\left(\theta_{20}\right) t+b\left(\theta_{20}\right)\left(\theta_{1}(t)-\theta_{10}\right) .
\end{aligned}
$$

Next, employing this corollary we show that the controllability Gramian of the SRC manipulator, as asserted by the general theory, drops its rank at the configuration singularities.

\section{Proposition 4 Let}

$\mathcal{M}_{q_{0}, T}(s(\cdot))=\int_{0}^{T} \Phi(T, t) B(t) B^{T}(t) \Phi^{T}(T, t) d t$,

where $q_{0}=\left(\theta_{10}, \theta_{20}, \phi_{0}\right)$, denote the controllability Gramian of system (27). Then, at either case 1 or 2 of Corollary 2

$\operatorname{rank} \mathcal{M}_{q_{0}, T}(s(\cdot))<3$.

Proof From expressions (27) and (6) we compute

$$
A(t)=\left[\begin{array}{ccc}
0 & 0 & 0 \\
0 & 0 & 0 \\
0 & a^{\prime}+b^{\prime} s_{1}+c^{\prime} s_{2} & 0
\end{array}\right], \quad B(t)=\left[\begin{array}{ll}
1 & 0 \\
0 & 1 \\
b & c
\end{array}\right],
$$


where ' refers to the derivative with respect to $\theta_{2}$ and all functions $a, b, c$ and their derivatives $a^{\prime}, b^{\prime}, c^{\prime}$ are evaluated at $\theta(t)=\theta_{20}$. Specifically, in case 1 this results in

$$
A(t)=\left[\begin{array}{ccc}
0 & 0 & 0 \\
0 & 0 & 0 \\
0 & a^{\prime}-b^{\prime} \frac{a}{b+1} & 0
\end{array}\right], \quad B(t)=\left[\begin{array}{ll}
1 & 0 \\
0 & 1 \\
b & c
\end{array}\right]=\text { const }
$$

But from definition (28) of $a$ and $b$, and since $F\left(\theta_{2}\right)=$ $I+G\left(\theta_{2}\right), G\left(\theta_{2}\right)=B+C+2 d \cos \theta_{2}$, it follows that $a^{\prime}-b^{\prime} \frac{a}{b+1}=0$. This being so, $A(t)=0, \Phi(t, s)=I_{3}$, and finally

$\operatorname{det}\left(\mathcal{M}_{q_{0}, T}(s(\cdot))=T B B^{T}\right)=0$.

Passing to case 2 we obtain

$$
A(t)=\left[\begin{array}{ccc}
0 & 0 & 0 \\
0 & 0 & 0 \\
0 & a^{\prime}+b^{\prime} s_{1} & 0
\end{array}\right], \quad B(t)=\left[\begin{array}{ll}
1 & 0 \\
0 & 1 \\
b & c
\end{array}\right]=\text { const }
$$

Referring to the definition of $a$ and $b$ we get $a^{\prime}=\frac{p F^{\prime}}{F^{2}}$ and $b^{\prime}=\frac{G F^{\prime}-G^{\prime} F}{F^{2}}$. But $F^{\prime}=G^{\prime}=2 D \sin \theta_{2}=0$, so $a^{\prime}=$ $b^{\prime}=0$, and again the matrix $A(t)=0$. By the same argument as above we conclude that

$\operatorname{det}\left(\mathcal{M}_{q_{0}, T}(s(\cdot))=T B B^{T}\right)=0$.

\section{Conclusion}

As mentioned in the Introduction, the characterization by normal forms of singularities of holonomic manipulators is known as the Normal Form Approach [1, 2]. In this paper, we extend this approach to non-holonomic robots. What we get are simple models of singularities, and for the reason that our feedback transformations can be computed explicitly, we also provide a description of configuration singularities and singular curves for the original system. We believe that the knowledge of singularities is not only interesting on its own, but also plays in the control of nonholonomic systems (in particular in Jacobian motion planning) exactly the same role as the knowledge of kinematic singularities in the control of holonomic manipulators. This topic will be investigated further in the future.

Specifically, using the normal forms, we have studied configuration singularities of non-holonomic robotic systems described by control-affine systems or driftless systems. An example of a space manipulator illustrates the applicability of the normal form approach. It may be expected that, besides characterizing the singularities, the feedback equivalence may also facilitate solving some control problems of the SRC space manipulator. This issue will be addressed in a separate paper.

Acknowledgments The authors are very much indebted to anonymous reviewers for their criticism that has allowed to improve both the contents as well as the presentation of this paper.

Open Access This article is distributed under the terms of the Creative Commons Attribution 4.0 International License (http:// creativecommons.org/licenses/by/4.0/), which permits unrestricted use, distribution, and reproduction in any medium, provided you give appropriate credit to the original author(s) and the source, provide a link to the Creative Commons license, and indicate if changes were made.

Publisher's Note Springer Nature remains neutral with regard to jurisdictional claims in published maps and institutional affiliations.

\section{References}

1. Tchoń, K., Muszyński, R.: Singularities of nonredundant robot kinematics. Int. J. Robot. Res. 16, 60-76 (1997)

2. Tchoń, K., Muszyński, R.: Singular inverse kinematic problem for robotic manipulators: a normal form approach. IEEE Trans. Robot. Autom. 14, 93-104 (1998)

3. Tchoń, K.: Endogenous configuration space approach: an intersection of robotics and control theory. In: Nonlinear Systems, pp. 209-234. Springer (2017)

4. Tchoń, K., Ratajczak, J.: Kinematic and dynamic singularities of non-holonomic robotic systems. In: Proc. 11th Int. Workshop RoMoCo, pp 148-153. Własowo Palace, Poland (2017)

5. Bonnard, B.: Feedback equivalence for nonlinear systems and the time optimal control problem. SIAM J. Control Optim. 29, 1300-1321 (1991)

6. Jakubczyk, B.: Critical hamiltonians and feedback invariants. In: Jakubczyk, B., Respondek, W. (eds.) Geometry of Feedback and Optimal Control, pp. 219-256. Marcel Dekker, New York-Basel (1998)

7. Respondek, W., Zhitomirski, M.: Feedback clasification of nonlinear systems on 3-manifolds. Math. Contr. Signals Syst. 8, 299-333 (1995)

8. Yoshida, K., Wilcox, B.: Space robots and systems. In: Springer Handbook of Robotics, pp. 1031-1063. Springer (2008)

9. Johnson, C.D., Gibson, J.E.: Singular solutions in problems of optimal control. IEEE Trans. Autom. Contr. 8(1), 4-15 (1963)

10. Bonnard, B., Chyba, M.: Singular Trajectories and Their Role in Control Theory. Springer, Paris (2003)

11. Chitour, Y., Jean, F., Trélat, E.: Singular trajectories of controlaffine systems. SIAM J. Contr. Opt. 47, 1078-1095 (2008)

12. L'Affito, A., Haddad, W.M.: Abnormal optimal trajectory planning of Multi-Body systems in the presence of holonomic and nonholonomic constraints. J. Intell. Robot. Syst. 89, 51-67 (2018)

13. Sontag, E.D.: Mathematical Control Theory. Springer, Berlin (1990)

14. Tchon, K., Ratajczak, J.: General Lagrange-type jacobian inverse for nonholonomic robotic systems. IEEE Trans. Robot. 34, 256263 (2018)

15. Rybus, T., Seweryn, K.: Planar air-bearing microgravity simulators: review of applications, existing solutions and design parameters. Acta Astronaut. 120, 239-259 (2016) 
16. Rybus, T.: Control of a Satellite Manipulator During the Interception Maneuver and the Motion Stabilization. Ph.D. dissertation, Wrocław University of Science and Technology (2016)

17. Zhitomirski, M., Respondek, W.: Simple germs of corank one distributions. In: Singularities Symposium, Banach Center Publications, vol. 44, pp. 259-266. Warsaw

18. Zhitomirskii, M.: Typical singularities of differential 1-Forms and pfaffian equations, Transl. Math Monographs 113. Amer. Math. Soc., Providence (1992)

Professor Krzysztof Tchoń received his Ph.D. degree in automation from Wroclaw University of Technology in 1976. In 1986 he got D.Sc. degree in control engineering and robotics from the same University. Since 1996 he has been a full professor of control engineering and robotics. He has been working at the Wroclaw University of Technology (now of Science and Technology) for 45 years. In the years 1986-2016 he chaired the Departmant of Cybernetics and Robotics at the Electronic Faculty of Wroclaw University of Science and Technology. His current reasearch interest concentrate on applications of mathematical methods in robotics, including motion planning, control, and singularity analysis of holonomic and nonholonomic robots. Professor K. Tchon has authored more than 200 publications, and promoted $13 \mathrm{PhD}$ students. He is a leader of the Seminar of Cybernetics and Robotics that started in 1973 and has had over 1200 meetings.
Professor Witold Respondek received his Ph.D. degree form the Institute of Mathematics, Polish Academy of Sciences in 1981. He has had positions at the Technical University of Warsaw and at the Polish Academy of Sciences. Since 1994 he has been a professor of applied mathematics at the INSA de Rouen, France. General areas of his scientific interest are geometric methods in systems and control theory as well as geometric methods in differential equations. His research papers have been devoted to problems of linearization of nonlinear control systems, nonlinear observers, classification of control systems and vector distributions, dynamic feedback, applications of highgain feedback to nonlinear systems, and systems invariant on cones. Recently he has been working on mechanical control systems and on flatness, with particular emphasis on systems with nonholonomic constraints, and on geometry of optimal control problems. $\mathrm{He}$ has been an associated editor of SIAM Journal on Control and Optimization, Applicationes Mathematicae, Central European Journal of Mathematics, and Journal of Geometric Mechanics.

Joanna Ratajczak received the M.S. and Ph.D. degrees in control engineering and robotics from the Wroclaw University of Science and Technology, Wroclaw, Poland in 2007 and 2012, respectively. She is currently an Assistant Professor at Wroclaw University of Science and Technology, Faculty of Electronics, Department of Cybernetics and Robotics. Her research interests include modelling, motion planning and control of holonomic and nonholonomic robots. 